\title{
Diesel PM collection for marine emission using hole-type electrostatic precipitators
}

\author{
Y. Ehara ${ }^{1}$, A. Osako ${ }^{1}$, A. Zukeran ${ }^{2}$, K. Kawakami ${ }^{3} \&$ T. Inui ${ }^{3}$ \\ ${ }^{I}$ Tokyo City University, Japan \\ ${ }^{2}$ Kanagawa Institute of Technology, Japan \\ ${ }^{3}$ Fuji Electric Co., Ltd., Japan
}

\begin{abstract}
The particulate matters (PMs) emitted from marine diesel engine exhaust during the combustion process have low resistivity and extremely small in the range of $70-120 \mathrm{~nm}$. These particles have various human health and environment impacts. After MARPOL 73/78 Annex VI entered into force on 1st of July 2010, shipping-induced NOx and SOx, PM emissions are regulated more strictly. The diesel particulate filter was widely used for the collection of automobile diesel PM but was not economical, especially for the use of marine engine emission control where PM concentration is as high as $50 \mathrm{mg} / \mathrm{m}^{3}$. Electrostatic Precipitators (ESPs) have been studied as an after treatment system for the removal of PM emissions from a diesel engine. However, the collection of low resistive particles using conventional ESPs is difficult. The low resistive diesel engine particles are detached from the collection plate, which causes particle reentrainment, resulting in poor collection efficiency. The new hole-type ESP was developed to minimize re-entrainment. The hole-type ESP utilizes the ionic wind, combined with electrostatic force to transport the charged particles effectively into the collecting zone through the hole of the collection plate. The collecting zones are designed as zero electrostatic fields, i.e., no electrostatic repulsion force acting on particles by induction charge, so that no re-entrainment takes place. The hole diameter with respect to aperture ratio influences the particle collection processes. The optimum hole diameter was determined by the time-dependent collection efficiency.

Keywords: particulate matters, diesel engine exhaust, electrostatic precipitator, re-entrainment.
\end{abstract}




\section{Introduction}

The particulate matters (PMs) emitted from diesel engine exhaust are low resistive in nature and extremely small in the range of $70 \sim 120 \mathrm{~nm}$. These particles cause a various human health and environment impacts. These particles are penetrated into an alveolus and extremely harmful to human health. These particles are generated from various emission sources such as diesel automobiles, marine engines, power generation engines, and construction machines. The particulate filter was widely used for the collection of automobile diesel PM but was not cost effective, especially for marine engine emission where PM concentration is often higher than $50 \mathrm{mg} / \mathrm{m}^{3}$. After MARPOL 73/78 Convention Revised Annex VI entered into force on 1st of July 2010, shipping-induced NOx and SOx, PM emissions are regulated stricter.

The collection of low resistive PM has been known to be extremely difficult by the conventional electrostatic precipitators (ESPs). The low resistive diesel engine particles are detached from the collection plate where the electrostatic repulsion force due to induction charge exceeds particle adhesion force on the collection electrode. This phenomenon has been known as particle reentrainment or resuspension, resulting in poor collection efficiency [1, 2]. Therefore, it is very important to prevent the particles from being re-entrained. Authors have been studying the technology of the re-entrainment inhibition in ESP. The particle re-entrainment was prevented by AC-ESP [3]. AC-ESP with hole-punched electrode was suggested to improve the nano particle collection efficiency and suppress particle re-entrainment [4].

Many methods were applied to control mass concentration of PM from diesel exhaust gas. Many studies of ESP were reported to collect low resistive particles generated from diesel engine $[5,6]$. Authors confirmed that a collection of PM was possible by a dielectric barrier discharge type ESP [7, 8]. Furthermore, a double cylinder type ESP is proposed to remove marine diesel exhaust particles [9]. The present major focus regarding double cylinder type ESPs is to study the numerical simulation for the aerodynamics behaviour and the re-entrained particle motion. The ESP consists of three electrodes, which are the high voltage saw electrode, grounded hole punched electrode and grounded electrode. Numerical simulation results show that re-entrainment of diesel particles can be suppressed with this double cylinder ESP configuration. Then, we decided to call this ESP a hole-type ESP.

This research has been developed an after treatment system for removal of PM emissions from a diesel engine. The new hole-type ESP, which has grounded hole plate electrode, was developed to overcome the re-entrainment and increase the collection efficiency. We investigated the effect of the aperture rate of the hole-punched electrode on improving the collection efficiency.

\section{Experimental setup}

A schematic diagram of the experimental setup for PM collection is shown in Fig. 1. Emissions from small diesel engine generator (Yammer Co., Ltd., 
YDG200A-5E, direct injection type for a single cylinder, displacement volume of $199 \mathrm{cc}$, maximum electric power output of $1.7 \mathrm{~kW}$ ) using light oil were used. The constituents of the diesel PM investigated were $99 \%$ of $\mathrm{C}, 0.1 \%$ of $\mathrm{Si}$, $0.07 \%$ of $\mathrm{Fe}, 0.1 \%$ of $\mathrm{Ca}, 0.4 \%$ of $\mathrm{S}$, and $0.03 \%$ of $\mathrm{Zn}$. In order to determine the number particle density in the ESP, the flue gas was diluted approximately 100 times by ambient air and particle size-dependent number densities before and after the ESPs were determined by the SMPS (Scanning Mobility Particle Sizer, Model 3080) for the particle size ranged 20-800 nm and the particle counter (Rion KC-01E) for the particle size of 300-5,000 nm, respectively. The exhaust gas temperature was nearly $130^{\circ} \mathrm{C}$. The gas velocity was $1.5 \mathrm{~m} / \mathrm{s}$. DC $-8 \mathrm{kV}$ was applied to the discharge electrode for 1 hour, and the discharge current was $0.5 \mathrm{~mA}$.

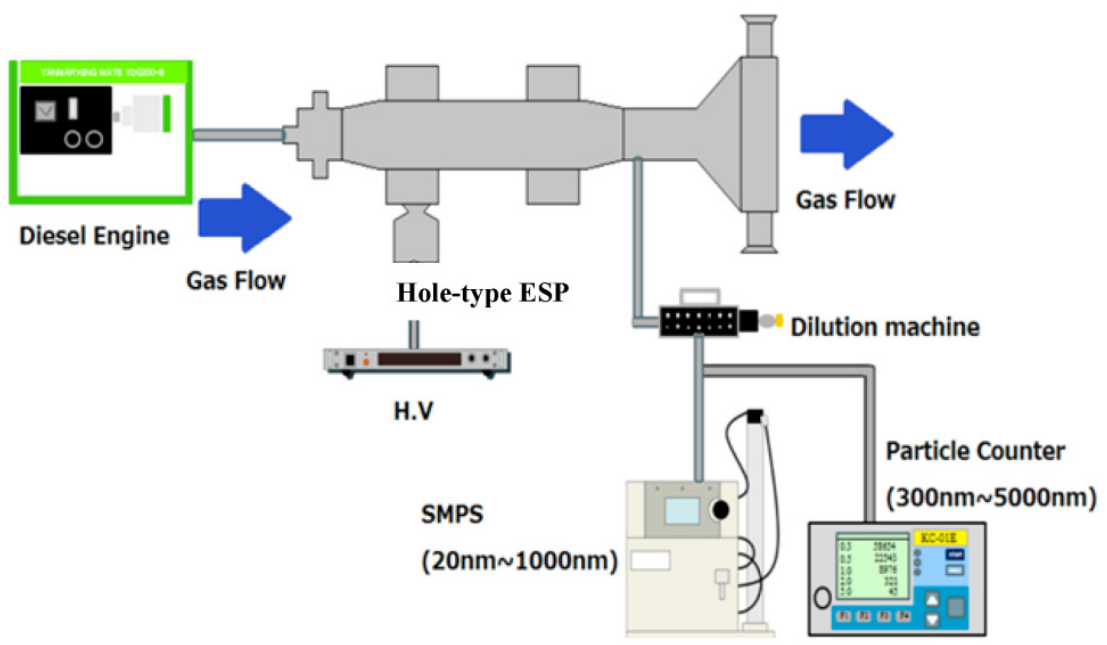

Figure 1: Experimental setup for PM collection.

The electrode structure of hole-type ESP used for this experiment was shown in Fig. 2, while the conventional ESP was the exactly the same as the hole-type ESP without holes. The hole-type ESP consists of the grounding case, the inner grounded hole electrode and the high voltage discharge electrode. Three kinds of hole diameters, $1 \mathrm{~mm}, 2.5 \mathrm{~mm}$, and $5 \mathrm{~mm}$, were used. Three kinds of aperture rates, $22.6 \%, 35.0 \%$, and $46.2 \%$, were used respectively. The upstream and downstream portions of the space between the hole electrode and the grounding case are closed. The flue gas is connected to the upstream portion between the discharge electrode and the hole electrode. A space between the grounded hole electrode and grounding case is collecting zone. The hole-type ESP utilizes the ionic wind, combined with electrostatic force to transport the charged particles effectively into the collecting zone through the hole of the collection plate. The collecting zones are designed as zero electrostatic field, i.e., no electrostatic repulsion force acting on particles by induction charge, so that no re-entrainment 


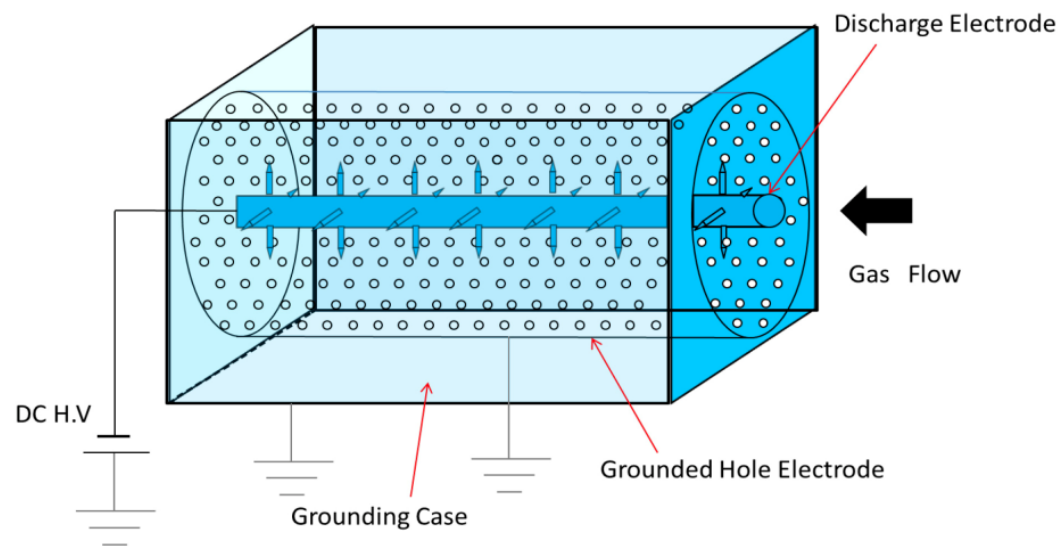

Figure 2: Electrode structure of hole-type ESP.

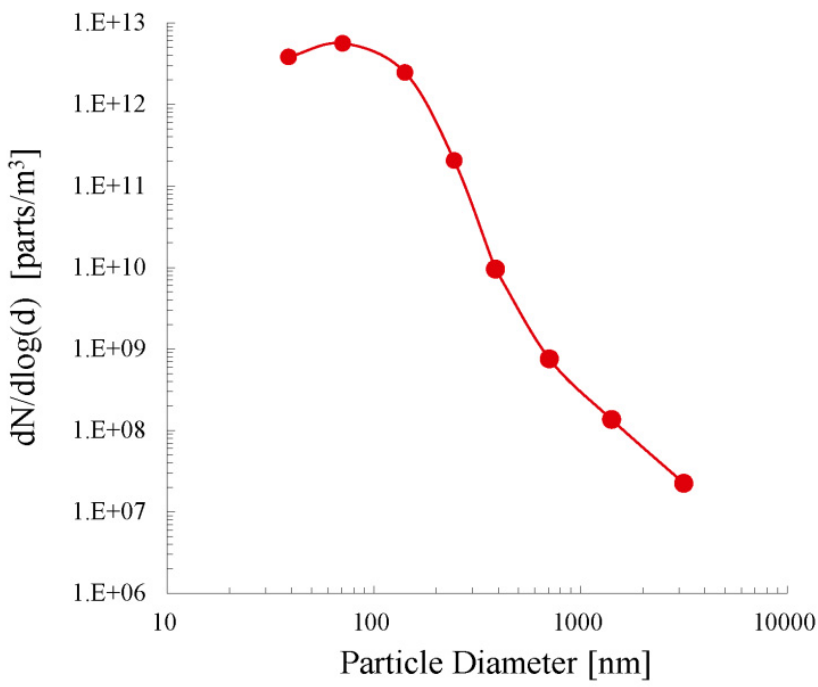

Figure 3: $\quad$ Particle size-dependent number density for $90 \%$ load upstream of hole-type ESP.

takes place. The inner grounded electrode of conventional ESP was the cylinderplate without the hole.

Fig. 3 shows the particle size distribution from the diesel engine particulates using the SMPS when the engine was $90 \%$ load $(1.5 \mathrm{~kW})$. As shown in Fig. 3 that the particle size was in the range of $10 \mathrm{~nm}$ and $350 \mathrm{~nm}$ in diameter with the peak number density of $5.5 \times 10^{12}$ parts $/ \mathrm{m}^{3}$ at $70 \mathrm{~nm}$ at the hole-type ESP upstream. It 
is clear that the ultra-fine particles, which are extremely harmful to human health, are included in the exhaust gas.

\section{Results and discussion}

Experiments were performed using 199cc diesel engine with light oil. When the engine load was set at $90 \%(1.5 \mathrm{~kW})$ with the gas flow velocity of $1.5 \mathrm{~m} / \mathrm{s}$, the particle-size dependent number density in the range of $20-5000 \mathrm{~nm}$ was evaluated using SMPS (20-500 nm) and PC (300-5000 nm) for the conventional ESP and hole-type ESP. Fig. 4 shows the particle-size dependent number collection efficiency for particle size of 20-500 nm measured by the SMPS and particle-size dependent number collection efficiency for 300-5000 nm measured by PC for the hole-type ESP and the conventional ESP without hole, when the discharge current was set at $0.5 \mathrm{~mA}$. The hole diameter of hole-type ESP was $1 \mathrm{~mm}$, aperture ratios were $22.6 \%, 35.05 \%, 46.2 \%$ respectively, and the inner grounded electrode of the conventional ESP was plate electrode. Clearly, the particle re-entrainment occurred for the conventional ESP and dominated with large particle size greater than $1000 \mathrm{~nm}$ as particles deposited on the collection plate which was relevant from the re-entrainment theory the conventional ESP [10]. On the other hand, the collection efficiency of an average of $90 \%$ was

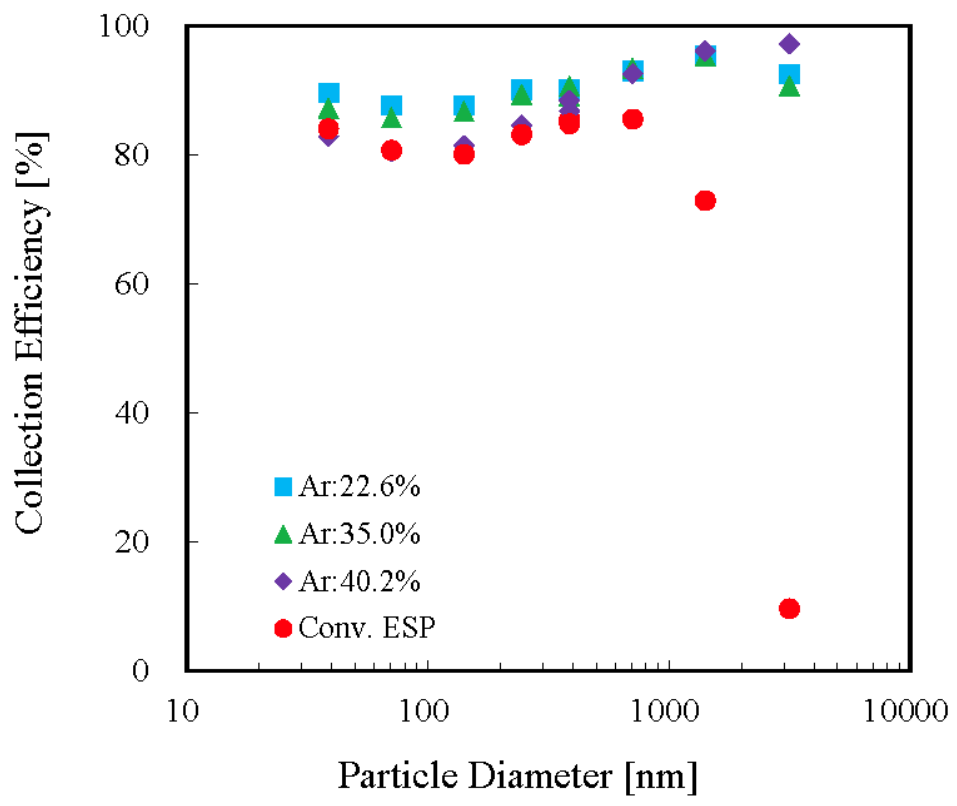

Figure 4: Particle-size dependent number density collection efficiency for the conventional ESP and the hole-type ESP with $1 \mathrm{~mm}$ hole diameter. 
achieved for the hole-type ESPs for particle size of $1000 \mathrm{~nm}$ or greater and particle re-entrainment was clearly suppressed. The collection efficiency between 20 to $1000 \mathrm{~nm}$ increases with decreasing the aperture ratio.

The particle-size dependent number density collection efficiency for the conventional ESP and the hole-type ESPs was measured by SMPS and PC as shown in Fig. 5. The hole diameter of hole-type ESPs was $5 \mathrm{~mm}$, aperture ratios were $22.6 \%, 35.0 \%, 46.2 \%$, respectively. The collection efficiencies for the holetype ESPs were superior for particle size of 20-1000 nm. However, the collection efficiencies for particle size of $1000-3000 \mathrm{~nm}$ decrease with increasing particle diameter, the particle re-entrainment occurred for each ESP.

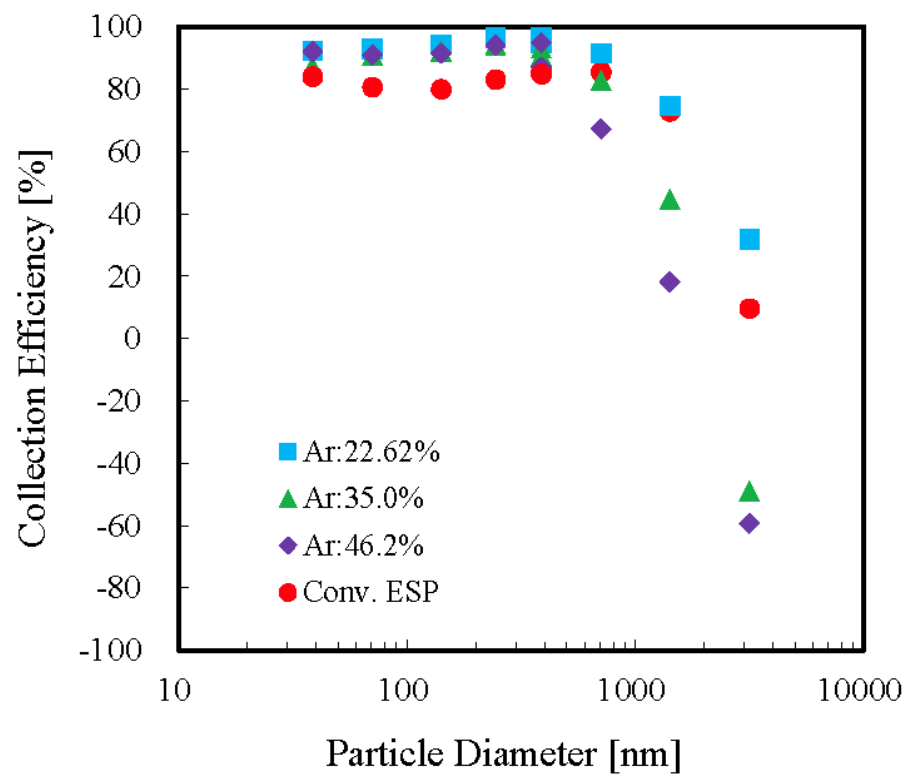

Figure 5: Particle-size dependent number density collection efficiency for the conventional ESP and the hole-type ESP with $5 \mathrm{~mm}$ hole diameter.

In order to understand the effects of the hole diameter for hole-type ESP, the overall number density collection efficiency was calculated in all the particle size. Fig. 6 shows the particle-size dependent the overall collection efficiency for the conventional ESP and the hole-type ESPs respectively. The overall collection efficiency in all the particle size for the conventional ESP was $81 \%$. It was shown that the hole-type ESPs are more overall collection efficiency than the conventional ESP. With the increase of aperture ratio, the overall collection efficiency for the hole-type ESP decreased obviously. In hole diameter with $2.5 \mathrm{~mm}$, the overall collection efficiency decreased from $88.4 \%$ to $80.7 \%$ when the aperture ratio increased from $22.6 \%$ to $46.2 \%$. The overall collection efficiency of more than $90 \%$ was achieved for the ESP of $5 \mathrm{~mm}$ hole diameter. 


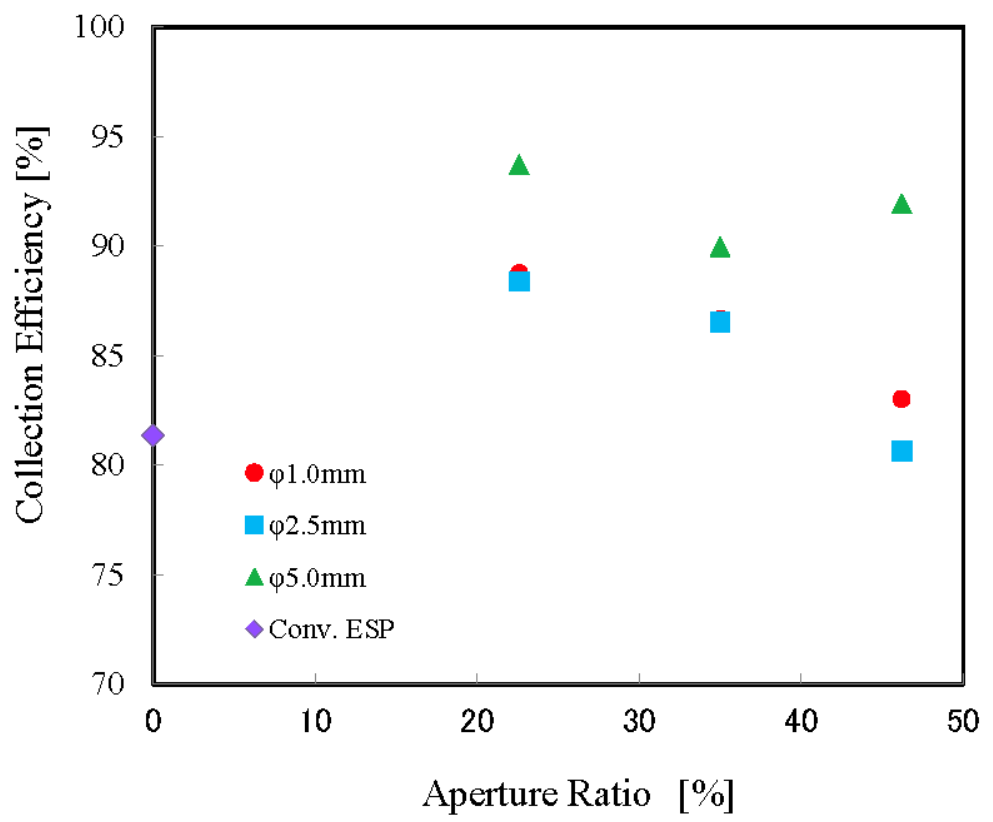

Figure 6: Effect of hole diameter on number density collection efficiency for hole-type ESP.

The particle-size dependents the overall mass collection efficiency for the conventional ESP and the hole-type ESPs were shown in Fig. 7. This figure shows the mass base collection efficiency for the conventional ESP which was $75.8 \%$. It was shown also in mass collection efficiency that the hole-type ESPs are better than the conventional ESP. With the increase of aperture ratio, the overall mass collection efficiency for the hole-type ESP decreased obviously. The mass base collection efficiency for the hole-type ESP with $5 \mathrm{~mm}$ hole diameter and $22.6 \%$ aperture ratio was the highest and was $92.2 \%$.

The time-dependent collection efficiency as a function of particle size of 300$5000 \mathrm{~nm}$ measured by the PC for the hole-type ESP with $1 \mathrm{~mm}$ hole diameter and $22.6 \%$ aperture ratio was shown in Fig. 8. The collection efficiency was increased with increased particle size and minimum for $300 \mathrm{~nm}$ range which implies the classical electrostatic charging theory, indicating no particle reentrainment occurred during 30 minutes operation. However, after passing for 30 minutes operation, the collection efficiency for particle size of $2000 \mathrm{~nm}$ or greater was decreasing gradually. In $1 \mathrm{~mm}$ with small hole diameter, it is considered that the holes are closed in deposition of the collected particles, and the collection efficiency decreases. This phenomenon is considered to be a sign of re-entrainment, since the collection efficiency of large particles is decreasing. 


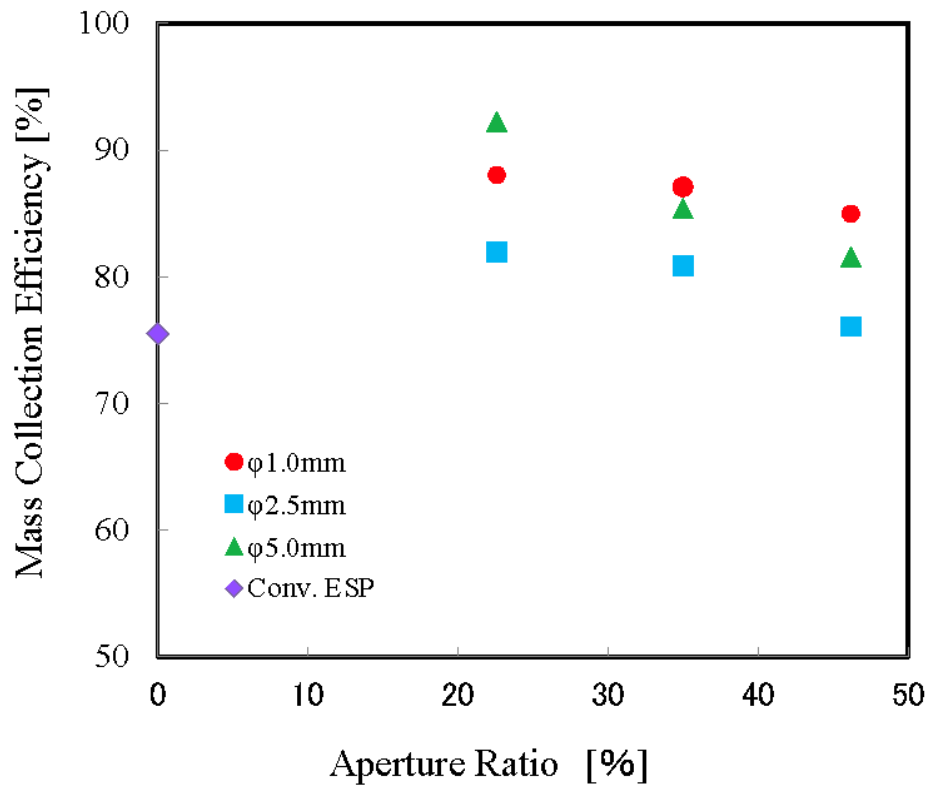

Figure 7: Effect of hole diameter on mass density collection efficiency for hole-type ESP.

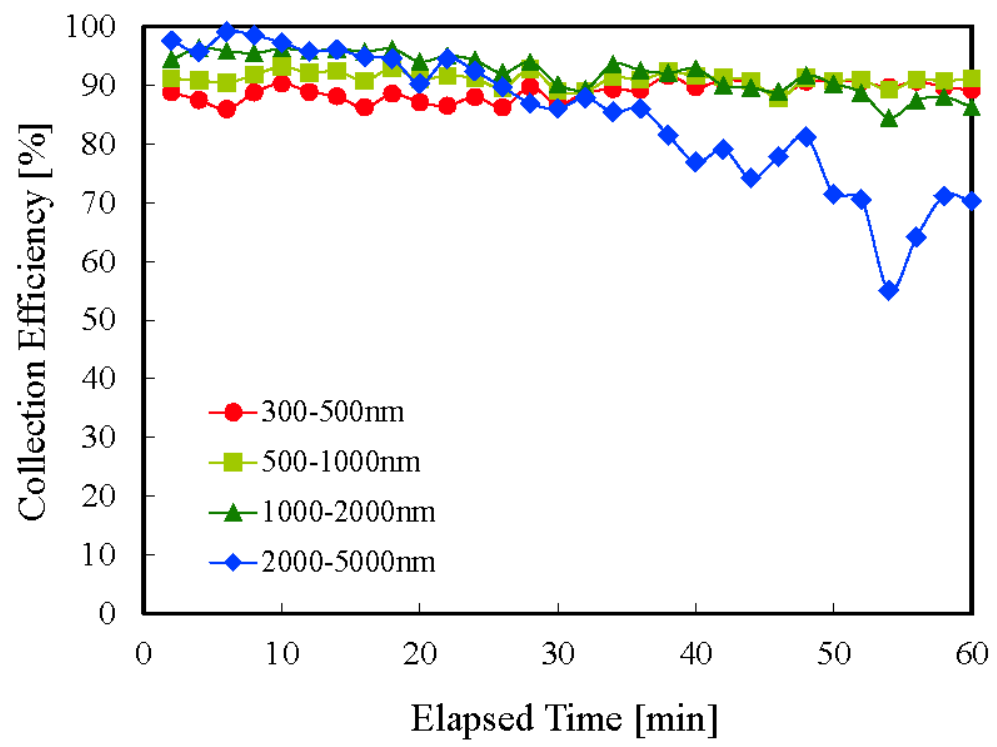

Figure 8: Time-dependent number density collection efficiency as a function of particle size of 300-5000 nm measured by the PC for the holetype ESP with $1 \mathrm{~mm}$ hole diameter and $22.6 \%$ aperture ratio. 
On the other hand, the time-dependent collection efficiency as a function of particle size of 300-5000 nm measured by the PC for the hole-type ESP with $5 \mathrm{~mm}$ hole diameter and $22.6 \%$ aperture ratio was shown in Fig. 9. More than $90 \%$ collection efficiency was achieved for particle-size range between 300 and $1000 \mathrm{~nm}$. The average collection efficiency for particle-size range between 1000 and $2000 \mathrm{~nm}$ was $80 \%$ and that for particle-size range over $2000 \mathrm{~nm}$ was $40 \%$. In $5 \mathrm{~mm}$ with a large hole diameter, it is considered that the holes are not easily closed by the collected particles, and the collection efficiency is stable.

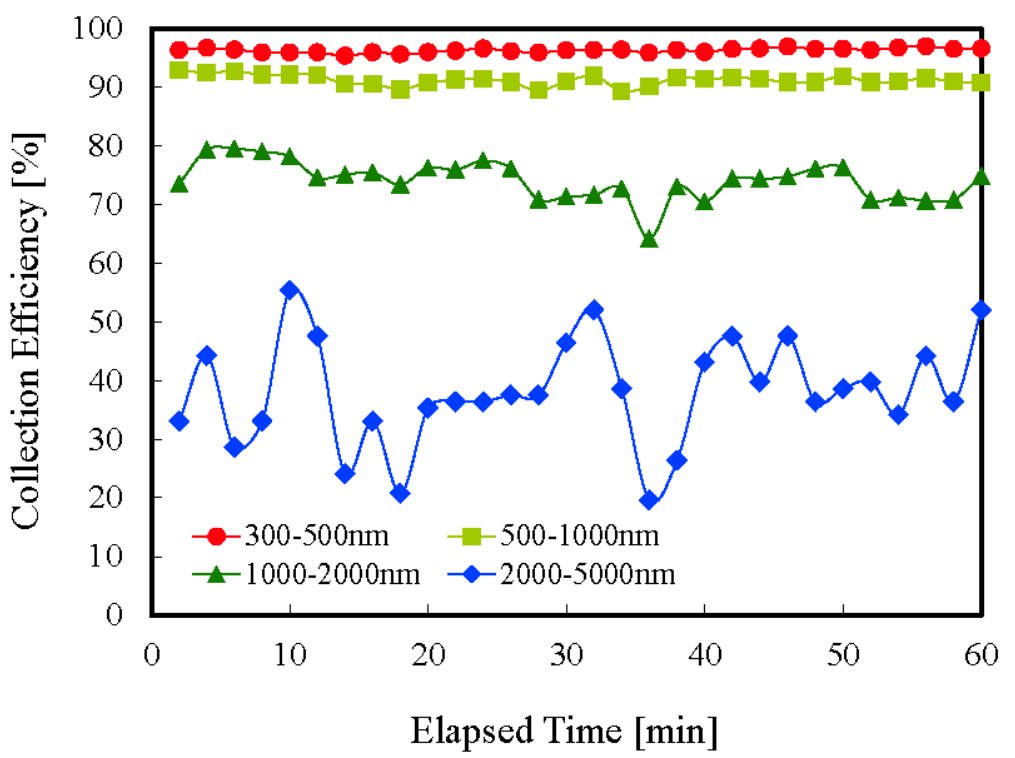

Figure 9: Time-dependent number density collection efficiency as a function of particle size of 300-5000 nm measured by the PC for the holetype ESP with $5 \mathrm{~mm}$ hole diameter and $22.6 \%$ aperture ratio.

Based on the collection efficiency for every hole diameter on the hole-type ESP, the hole-type ESP with $5 \mathrm{~mm}$ hole diameter performed better especially the particle size of 300-1000 nm range.

\section{Summary}

The collection of low resistive particles generated from diesel engine was investigated using the hole-type ESP. The conventional ESP showed good collection efficiency for particle size less than $1000 \mathrm{~nm}$ where adhesion force was dominated over electrostatic repulsion force but showed severe reentrainment for the particle size greater than 1,000 $\mathrm{nm}$. The hole-type ESPs showed good collection efficiency for the whole particle size ranging 20$5000 \mathrm{~nm}$. On the other hand, the hole-type ESP with the $22.6 \%$ aperture ratio 
showed an excellent collection efficiency for the entire particle size range investigated and the particle re-entrainment totally suppressed for larger particles. The hole-type ESP has high potential especially for highly concentrated marine diesel engine emission control.

\section{Acknowledgement}

The authors wish to express their thanks for the support by Grant-in-Aid for Scientific Research (B) of the Japanese Society for the Promotion of Science.

\section{References}

[1] J.D. Bassett, K. Akutsu, S. and Masuda, "A Preliminary Study of Reentrainment in an Electrostatic Precipitator", Journal of Electrostatics, Vol. 3, pp. 311-257, 1977.

[2] S. Masuda, J.D. Moon, K. Aoi, “AUT-AINER Precipitator System - an Effective Control Means for Diesel Engine Particulates", Actas 5, Congreso Int Aire Pure 1980 Tomo 2, pp. 1149-1153, 1982.

[3] A. Zukeran, Y. Ikeda, Y. Ehara, M. Matsuyama, T. Ito, T. Takahashi, H. Kawakami, and T. Takamatsu, "Two-Stage Type Electrostatic Precipitator Re-entrainment Phenomena under Diesel Flue gases," IEEE Trans. Ind. Applications, Vol. 35, No. 2, pp. 346-351, 1999.

[4] K. Yasumoto, A. Zukeran, Y. Takagi, Y. Ehara, T. Yamamoto, "Improving nano-particle collection efficiency and suppressing particle reentrainment in an AC electrostatic precipitator with hole-punched electrode," 11th International Conference on Electrostatic Precipitation pp. 251-255, 2008.

[5] S. Masuda, "Electrostatic Precipitation of Carbon Soot from Diesel Engine Exhaust", IEEE Trans. on Industry Applications Vol. IA 19, No. 6, pp. 1104-1111, 1983.

[6] M. Higashi, S. Uchida, N. Suzuki, and K. Fujii, "Soot Elimination and $\mathrm{NO}$, and SO, Reduction in Diesel-Engine Exhaust by a Combination of Discharge Plasma and Oil Dynamics," IEEE Trans. on Plasma Science, vol. 20, no. 1, pp. 1-12, 1992.

[7] Y. Kuroda, Y. Kawada, T. Takahashi, Y. Ehara, T. Ito, A. Zukeran, Y. Kono, K. Yasumoto. "Effect of Electrode Shape on Discharge Current and Performance with Barrier Discharge type Electrostatic Precipitator," Journal of Electrostatics, Volume 57, pp. 407-415, 2003.

[8] Y. Kuroda, Y. Kawada, T. Takahashi, Y. Ehara, T. Ito, A. Zukeran, Y. Kono, K. Yasumoto. "Effect of Metal Electrode Shape on Discharge Current and Performance in Barrier Discharge Type Electrostatic Precipitator," Journal of the Institute of Electrostatics Japan, Vol. 27, No. 3, pp. 146-147, 2003.

[9] H. Kawakami, A. Zukeran, K. Yasumoto, T. Inui, Y. Enami, Y. Ehara, T. Yamamoto, "Numerical Simulation of Re-Entrained Particle Migration for 
Double Cylinder Type Electrostatic Precipitator", Journal of the Japan Institute of Marine Engineering, Vol. 46, No. 5, pp. 769-776, 2011.

[10] K. Yasumoto, A. Zukeran, Y. Takagi, Y. Ehara, T. Takahashi, T. Yamamoto, "Suppression of particle deposition onto downstream wall in an AC Electrostatic Precipitator with neutralisation", International Journal of Environment and Waste Management, Vol. 2, No. 4-5 pp. 399-411, 2008. 